\title{
Coupling of plasmonic and optical cavity modes in quasi-three-dimensional plasmonic crystals
}

Debashis Chanda', Kazuki Shigeta', Tu Truong1', Eric Lui', Agustin Mihi', Matthew Schulmerich², Paul V. Braun', Rohit Bhargava² \& John A. Rogers ${ }^{1,3}$

The field of plasmonics has emerged as an interesting area for fundamental studies, with important application possibilities in miniaturized photonic components. Plasmonic crystals are of particular relevance because of large field enhancements and extraordinary transmission that arise from plasmonic interactions between periodic arrays of metallic elements. Here we report methods to enhance and modify the plasmonic resonances in such structures by strongly coupling them to optical modes of Fabry-Perot type cavities. First, we illustrate a type of plasmonic, narrow-band $(\sim 15 \mathrm{~nm})$, high-contrast ( $>20 \mathrm{~dB})$ absorber and an opto-fluidic modulator based on this component. Second, we use optimized samples as substrates to achieve strong amplification ( $>350 \%$ ) and modulation ( $>4 \times$ ) of surface-enhanced Raman scattering from surface-bound monolayers. Cavity-coupling strategies appear to be useful not only in these two examples, but also in applications of plasmonics for optoelectronics, photovoltaics and related technologies.

\footnotetext{
1 Departments of Materials Science and Engineering, Beckman Institute, and Frederick Seitz Materials Research Laboratory, University of Illinois at Urbana-Champaign, Urbana, Illinois 61801, USA. ${ }^{2}$ Department of Bioengineering, University of Illinois at Urbana-Champaign, Urbana, Illinois 61801, USA. ${ }^{3}$ Department of Mechanical Science and Engineering, University of Illinois at Urbana-Champaign, Urbana, Illinois 61801, USA. Correspondence and requests for materials should be addressed to J.A.R. (email: jrogers@uiuc.edu).
} 
$\mathrm{S}$ urface plasmons are coherent oscillations of electrons at metal-dielectric interfaces that can exist either in localized or propagating forms ${ }^{1}$. Such electromagnetic disturbances can be exploited for wide-ranging applications, from biological and chemical sensors based on changes in near-surface refractive index or Raman scattering ${ }^{2}$, to devices for sub-wavelength manipulation of light $^{3}$, wave-guiding ${ }^{4}$, optical modulation ${ }^{5}$, Bragg reflection ${ }^{6}$, photo detection $^{7,8}$, lasers ${ }^{9}$ and solar energy generation ${ }^{10}$. Methods to modify plasmonic interactions by controlling the shapes and sizes of the metal structures, ${ }^{11,12}$ the types of metals ${ }^{13,14}$ or the indices of refraction of the surrounding materials ${ }^{2,15}$ are firmly established. Newer approaches integrate plasmonic components into equivalent circuit structures such as metal-insulator-metal stacks ${ }^{16-18}$.

Here we report a different strategy in which light transmitted through a quasi-three-dimensional (quasi-3D) plasmonic crystal strongly couples with an underlying, Fabry-Perot cavity. We show that, in certain configurations, this arrangement leads to strongly enhanced or diminished reflections ( $\sim 23 \mathrm{~dB}$ change), due to significant modifications in the nature of the plasmonic modes, in a way that can be tuned reversibly using the techniques of opto-fluidics. Associated amplification in the surface plasmon field can also be exploited, to increase signal levels in surface-enhanced Raman scattering (SERS) experiments, by up to $\sim 350 \%$ and to modulate SERS signals $(>4 \times)$ in ways that are not possible with other known techniques. We capture all of these effects in a quantitative way by finitedifference time-domain (FDTD) electromagnetic modelling and systematic experiments. The design possibilities enabled by these ideas, especially when implemented using simple, large-area softimprint lithography techniques, lead to new classes of photonic/ plasmonic components.

\section{Results}

Cavity-coupled plasmonic crystal system. The structures are formed by first imprinting a square array of sub-wavelength cylindrical depressions ${ }^{19-21}$ on the surface of a thin polymer film cast on a glass substrate coated with a layer of gold. Blanket deposition of a thin layer of gold on the imprinted surface of the polymer completes the fabrication. Here a two-dimensional plasmonic crystal, formed by the top gold coating, optically couples to an array of isolated disks of gold in the bottoms of the cylindrical depressions. Light transmitted through this 'quasi-3D' crystal via plasmonic coupling between the nanoholes and recessed disks ${ }^{22}$ coherently reflects from the back mirror (that is, the layer of gold on glass) which in turn, influences the plasmonic resonances, constructively or destructively depending on the cavity length.

Figure 1 shows a large area $(5 \mathrm{~mm} \times 5 \mathrm{~mm})$ optical image (Fig. 1a) and a schematic illustration (Fig. 1b) of the plasmonic crystaloptical cavity system. A scanning electron microscope (SEM) top view image appears in Figure 1c, along with a magnified view of an individual nanohole/nanodisk (Fig. 1d). Figure 1e presents a cross-sectional SEM image and a schematic illustration (Fig. 1f) of a unit cell, showing the top gold film with a square lattice of circular holes, recessed circular gold disks (with some gold on the sidewalls near the base) and the gold back reflector $\left(H_{\mathrm{BR}}\right)$. The choice of nanohole period, $P=740 \mathrm{~nm}$, and diameter, $D=500 \mathrm{~nm}$, maximizes the strengths of localized surface plasmon resonances (LSPRs) of interest for present purposes ${ }^{20}$. This system embeds two asymmetric Fabry-Perot cavities, both with the thick gold as a back reflector: one formed by the gold in the raised regions of the top surface (Cavity (I)) and the other by the gold in the recessed regions (Cavity (II)). The lengths of these two cavities are related by the depth of moulded relief $\left(H_{\mathrm{RD}}\right)$, as shown in Figure 1f.

The geometries of the depressions, the thicknesses of the gold layers and the indices of the surrounding dielectric media (air and polymer) define the wavelengths of the LSPRs. The optical cavities strongly modify these resonances. Figure $2 \mathrm{a}$ shows the far-field
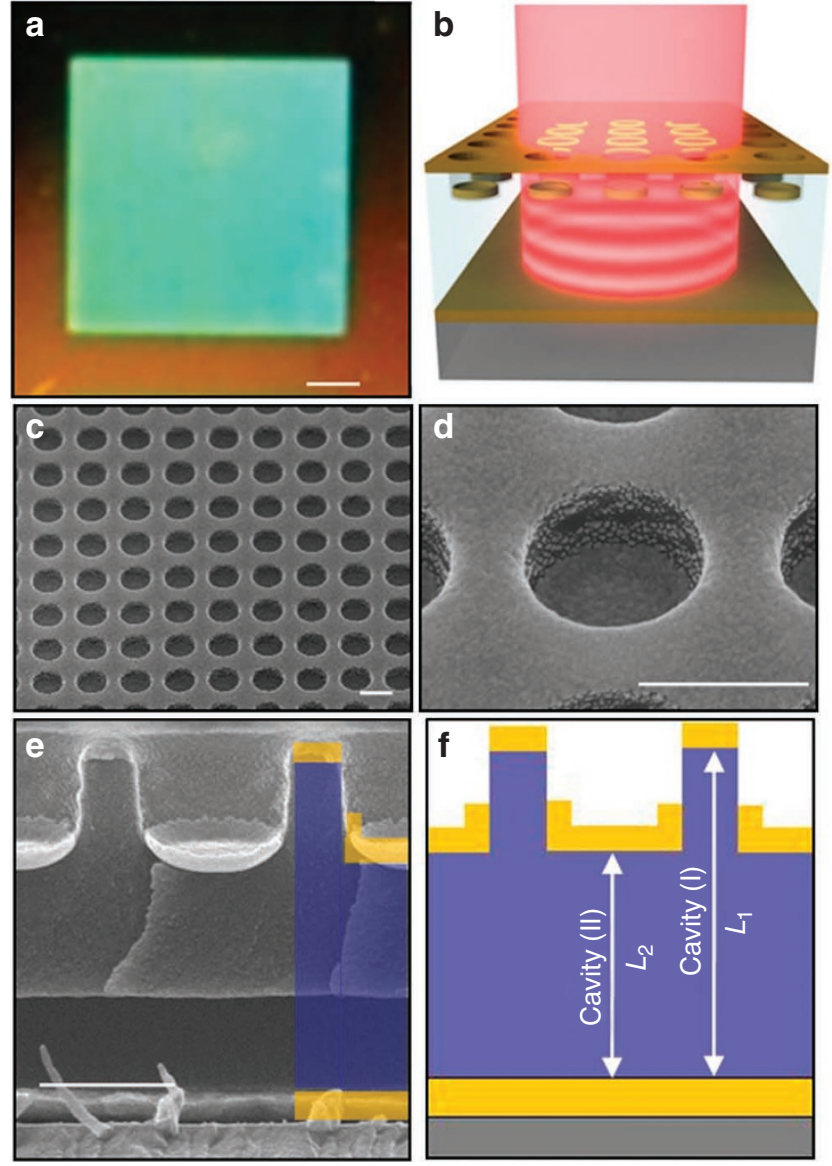

Figure 1 | Cavity-coupled quasi-3D plasmonic crystal. Large area optical image (scale bar $1 \mathrm{~mm}$ ) (a) and schematic illustration (b) of a cavitycoupled, quasi-3D plasmonic crystal. Top view SEM (c) of a small region of the sample shown in (a), with magnified view of a unit cell (d). Crosssectional SEM (e) and schematic illustration (f) of a unit cell showing gold on the raised and recessed regions of the plasmonic crystals, and the gold back reflector for the coupled optical cavities (lengths, $L_{2}$ and $L_{1}$, as in (e)). Scale bar $500 \mathrm{~nm}$.

reflectivity spectra measured experimentally and computed by FDTD, for a cavity-coupled plasmonic crystal with $L_{1}=1,170 \mathrm{~nm}$ and $H_{\mathrm{BR}}=200 \mathrm{~nm}$, along with FDTD results for a case without the cavity (that is, $H_{\mathrm{BR}}=0 \mathrm{~nm}$ ). The cavity clearly enhances the LSPR resonance that appears in the wavelength range $780-900 \mathrm{~nm}$, as manifested by a strong, sharp dip in the reflection at $\sim 845 \mathrm{~nm}$. The long-wavelength region $(1.5-6 \mu \mathrm{m})$, which lies away from the plasmonic resonances, shows unmodulated cavity modes, due to the combined effects of Cavity (I) and Cavity (II) (Supplementary Fig. S1). To reveal further features, Figure $2 \mathrm{~b}$ shows computed variations in the electric field intensity $\left(|\bar{E}|^{2}\right)$ spatially averaged across a conformal surface that extends over a unit cell of the plasmonic crystal at a position $10 \mathrm{~nm}$ above the raised and recessed regions of the gold layers, as a function of cavity length $\left(L_{1}\right)$ and the excitation wavelength, with all other structure parameters fixed, except for $L_{2}$, which changes according to $L_{2}=L_{1}-H_{\mathrm{RD}}$. The results show that the plasmon resonances bend and repeat with changes in $L_{1}$, as they come into and out of overlap with the cavity modes (Supplementary Figs S2 and S3). As might be expected, the cavity length and refractive index of the dielectric layer influence the effective optical-path lengths and, therefore, the positions of the cavity-mode resonances. By contrast, the index of the surroundings predominantly affects only the plasmonic component of the responses. Figure $2 c$ shows the FDTD-computed variation of the far-field reflection as a function of this index, for a struc- 

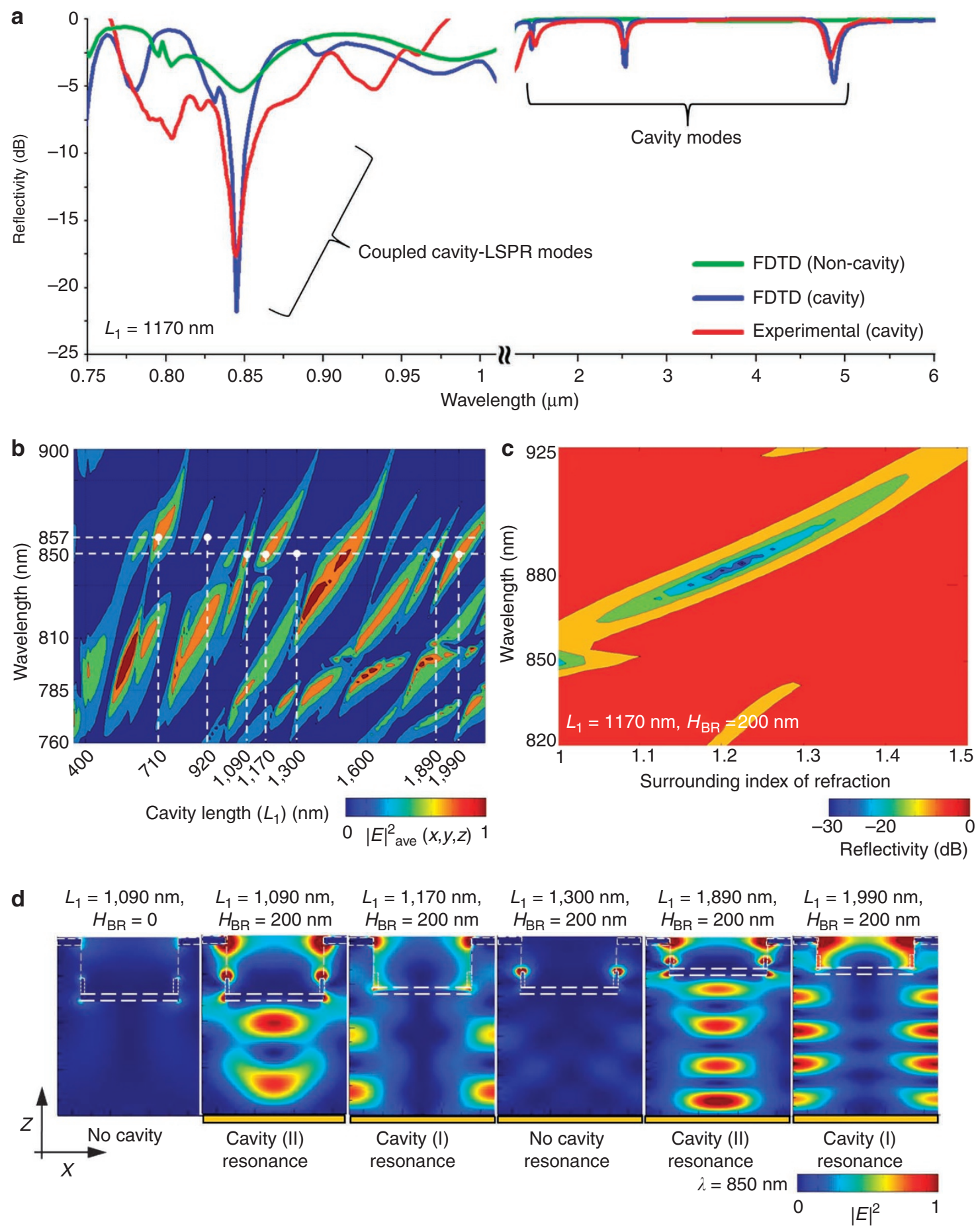

Figure 2 | Measured and computed properties in a cavity-coupled quasi-3D plasmonic crystal. (a) Measured and FDTD-computed far-field reflectivity of a cavity-coupled plasmonic crystal system having cavity length $L_{1}=1,170 \mathrm{~nm}$ with a thick back reflector $H_{B R}=200 \mathrm{~nm}$. Calculation for the case of $H_{B R}=0 \mathrm{~nm}$ is also shown. (b) FDTD-computed variation of the intensity $\left(|\bar{E}|^{2}\right)$, spatially averaged across a conformal surface located $10 \mathrm{~nm}$ above the top and bottom Au layers, as a function of cavity length $\left(L_{1}\right)$ and wavelength. (c) FDTD-computed variation of the far-field reflectivity as a function of surrounding medium refractive index for $L_{1}=1,170 \mathrm{~nm}$. (d) FDTD-computed cross-sectional $(X-Z)$ intensity distributions across a unit cell for various configurations. The results clearly show the influence of both cavities in this system.

ture with $L_{1}=1,170 \mathrm{~nm}$. Here the LSPR, initially near $850 \mathrm{~nm}$, moves to higher wavelengths with increasing index, hence reducing field enhancements due to de-tuning away from the cavity resonance. The phenomenon repeats because of overlap between the shifted LSPR and another cavity mode at an index of $\sim 1.22$ at a wavelength $\sim 885 \mathrm{~nm}$. The nature of the coupling is clearly visible in the cross-sectional intensity distributions of Figure $2 \mathrm{~d}$, computed using
FDTD for linearly polarized light at a wavelength of $850 \mathrm{~nm}$ for air $(n=1)$ as the surrounding medium. Specifically, the main plasmonic resonance is strongly enhanced when $L_{1}$ is 1,170 or $1,990 \mathrm{~nm}$ and when $L_{1}$ is 1,090 or $1,890 \mathrm{~nm}$, due to the presence of standing waves (that is, cavity modes) corresponding to the action of Cavity (I) and Cavity (II), respectively. For reference, when $L_{1}=1,300 \mathrm{~nm}$, no cavity mode is present and no enhancement results. The 
cross-sectional intensity distribution for $H_{\mathrm{BR}}=0$ (that is, non-cavity case) at $L_{1}=1,090 \mathrm{~nm}$ provides further evidence of the importance of the cavity (Supplementary Fig. S2).

Narrow-band plasmonic absorber/filter and opto-fluidic modulator. There are several ways to exploit the behaviours illustrated in Figure 2. The physics that governs the responses of these structures is different than that associated with absorbers that use other types of plasmonic structures and metamaterials ${ }^{23-25}$. The remarkably strong absorption and narrow-band operation reported here represent appealing features for applications in tunable filters, substrates for SERS, and other devices, as described in the following. In a first example, we demonstrate a class of narrow-band absorber, or reflective notch filter, and a modulator based on this component. The underlying effect is that light can be either nearly completely absorbed or completely reflected, depending on cavity lengths, by virtue of cavity controlled excitation of LSPRs on the gold surfaces. Figure $3 \mathrm{a}$ shows the variation of far-field reflection as a function of wavelength for $L_{1}=710 \mathrm{~nm}$ and $920 \mathrm{~nm}$. The former configuration results in an enhanced plasmonic resonance and low far-field reflection, which we label as the 'Absorption ON' state. The latter corresponds to a suppressed plasmonic resonance and high far-field reflection, that is 'Absorption OFF'. Coupling between LSPR modes and standing waves in the Fabry-Perot cavity originates from coherent interference effects. When the cavity produces constructive (destructive) interference near the surface of the structure, the fields available to excite LSPR modes are maximized (minimized), and the resonance is thereby enhanced (suppressed). For example, when $L_{1}=710 \mathrm{~nm}$, the round trip phase delay from propagation in the cavity is $\sim 4 \pi$ for the wavelength, $\lambda=857 \mathrm{~nm}$, that coincides with the main LSPR. In this scenario, constructive interference occurs, thereby enhancing the LSPR. Similarly, for $L_{1}=920 \mathrm{~nm}$, the phase delay is $\sim 5 \pi$, destructive interference obtains, and the LSPR is suppressed. These outcomes are apparent in the results of Figure 3a, where the transmission dip associated with the LSPR is large and small for $L_{1}=710 \mathrm{~nm}$ and $920 \mathrm{~nm}$, respectively. The measured bandwidth ( $15 \mathrm{~nm}, \mathrm{FWHM})$ and depth of the resonant dip in reflection at $\lambda=857 \mathrm{~nm}$ closely match FDTD predictions. Figure $3 \mathrm{~b}$ shows top $(\mathrm{X}-\mathrm{Y})$ and cross-sectional $(\mathrm{X}-\mathrm{Z})$ views of the intensity distributions. The 'Absorption ON' case shows a complex coupled Cavity (II) type mode, whereas the 'Absorption OFF' configuration indicates an absence of cavity effects. These two states also appear in characteristic features of Figure $2 b$ (white vertical dashed lines on the left, at $L_{1}=710$ and $920 \mathrm{~nm}$ ). This structure, then, represents an unusual optical component, with absorption properties that can be designed by appropriate choice of cavity dimensions. The position of the plasmonic absorption band depends strongly on the geometry of the plasmonic crystal (for example, P, D) ${ }^{9}$ and the intrinsic properties of the gold. Alternative metals can provide further flexibility in design ${ }^{13}$. When viewed as a reflective filter, the extinction coefficient $(>-25 \mathrm{~dB})$ and bandwidth $(\sim 15 \mathrm{~nm})$ of this three-layer plasmonic device are comparable to parameters that require, in a multilayer dielectric thin film Bragg structures, at least 20 alternating quarter-wave stacks with low index contrast $(\Delta n \sim 0.05-0.1)$ plus a half-wave defect layer followed by another 20-layer quarter wave $\operatorname{stack}^{26}$.

An extra attractive aspect of this type of cavity-coupled plasmonic component is that its properties can be tuned, dynamically, by changing either the dimensions or index of refraction of the cavity. For example, adjusting the cavity index by amounts $(\sim 5-10 \%)$ achievable with organic electro-optic materials ${ }^{27}$ can accomplish complete switching (Supplementary Fig. S4). An alternative, and simpler, mode of tuning involves changing the surrounding refractive index, using effects summarized in Figure 2c. Figure 4a shows, as a demonstration, reflection spectra collected from a sample with $L_{1}=710 \mathrm{~nm}$, switched between $\mathrm{ON}(-25 \mathrm{~dB})$ and OFF $(2 \mathrm{~dB})$
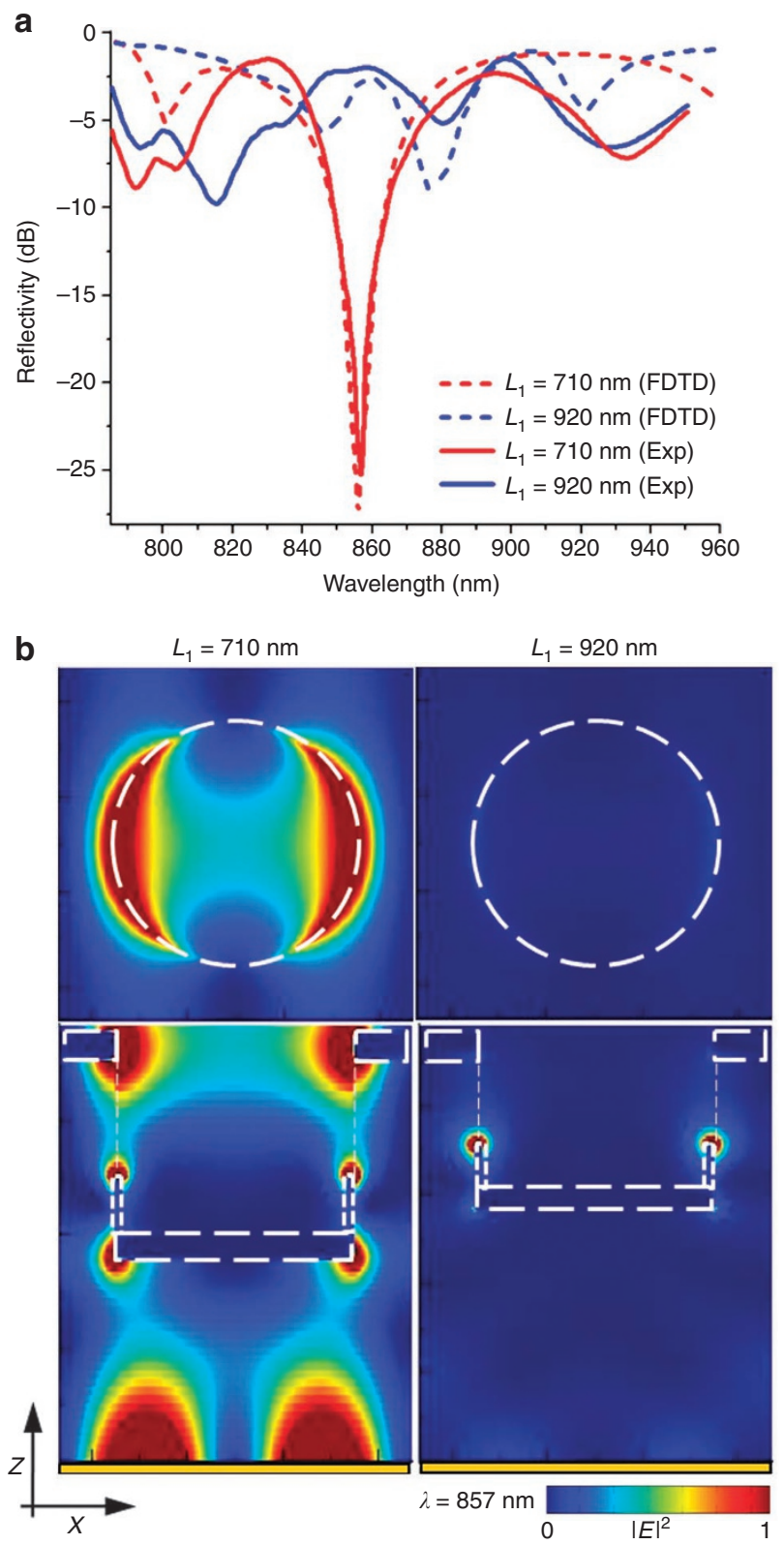

Figure 3 | Cavity controlled narrow-band absorption in a plasmonic crystal. (a) Far-field reflection as a function of wavelength measured and computed (FDTD) from structures for cavity lengths of $L_{1}=710 \mathrm{~nm}$ (Absorption ON) and $L_{1}=920 \mathrm{~nm}$ (Absorption OFF), both with a back reflector thickness of $H_{B R}=200 \mathrm{~nm}$. (b) FDTD-computed top $(X-Y)$ and cross-sectional $(X-Z)$ intensity distributions for these two cases, at $\lambda=857 \mathrm{~nm}$.

states at $\lambda_{0}=857 \mathrm{~nm}$ by replacing the surrounding air with ethanol $(n=1.3614)$. The techniques of opto-fluidics allow this type of switching to be achieved in an integrated manner. Figure $4 \mathrm{~b}$ shows a schematic illustration (left) and picture (right) of a device consisting of micro channel created by the contact of an embossed elastomer against a substrate that supports a cavity-coupled plasmonic structure. Optical images of this plasmonic crystal system in air and immersed in ethanol, collected in reflection mode through a narrow band bandpass filter (815-885 nm), appear in Figure 4c. Significant changes in brightness correspond to large differences in reflectivity between the air and ethanol cases. These changes occur uniformly over the entire imaged area, as expected based on the high quality 
a
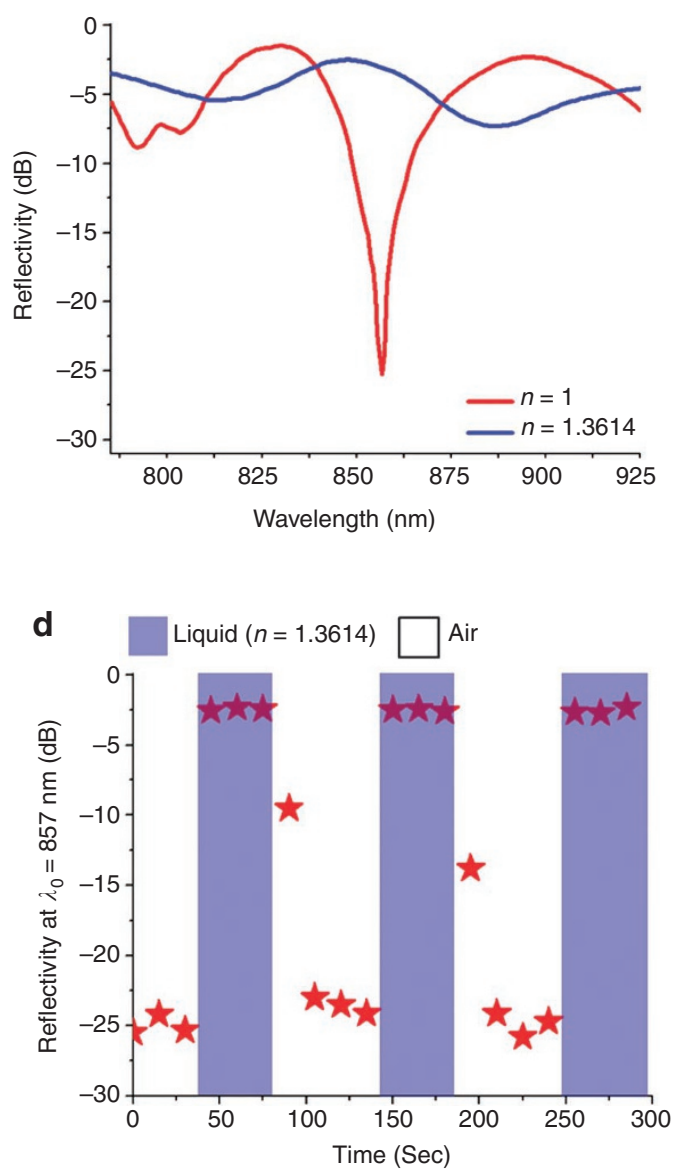

b

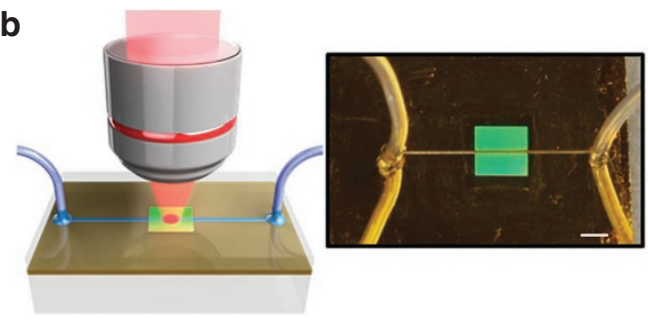

C

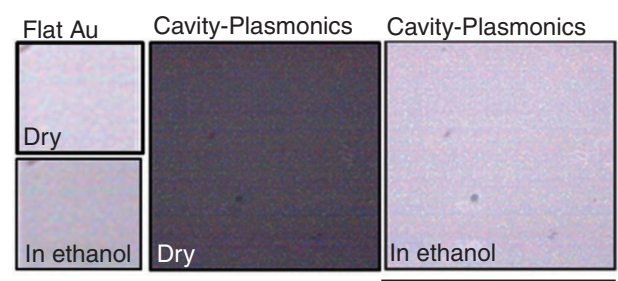

e

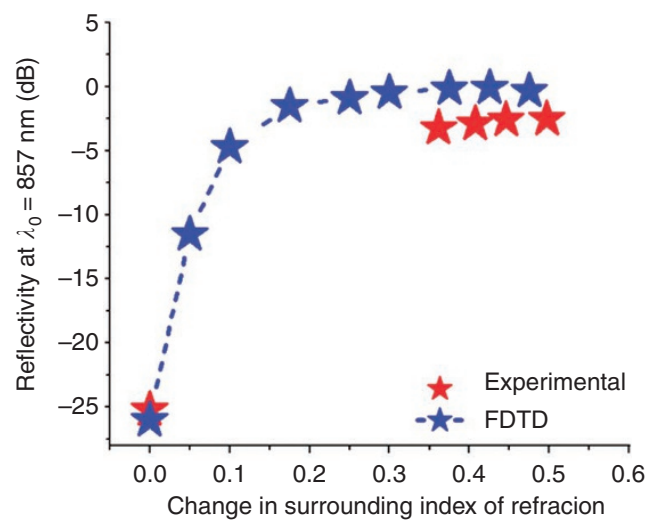

Figure 4 | Narrow band plasmonic absorber/filter and opto-fluidic modulator. (a) Switching 'ON' and 'OFF' of reflectivity due to shifts in the LSPR by a contacting liquid ( $n=1.36$ ) on the top gold surface. (b) Moulded Opto-fluidic chip mounted on a cavity-coupled plasmonic crystal; (left) schematic and (right) actual fabricated system. (c) Optical images of this cavity-coupled plasmonic crystal system in air and immersed in ethanol collected in reflection mode through a narrow band bandpass filter $(815-885 \mathrm{~nm})$. (d) Opto-fluidic switch showing a large change in reflectivity; measured reflectivity as a function of refractive index at $\lambda_{0}=857 \mathrm{~nm}$ is shown in (e). Scale bar $2.5 \mathrm{~mm}$.

of samples formed by soft imprint lithography procedures. Using a syringe pump, the surrounding material can be switched from ethanol to air, and back again, in a reversible manner. Here the LSPR, initially near $857 \mathrm{~nm}$, moves to higher wavelengths because of the increased index of the surroundings. As a result, the LSPR and cavity modes are no longer in resonance, thereby eliminating the enhancement effects. The process operates in a reversible manner such that the reflectivity goes down to its original value as the surrounding medium is switched from ethanol back to air, as shown in Figure $4 \mathrm{~d}$. The same channel system allows more systematic study with different liquids having refractive indices between 1.36-1.5. The change in reflectivity at $\lambda_{0}=857 \mathrm{~nm}$ as a function of refractive index appears in Figure 4e; FDTD results indicate a linear variation with index up to $\sim \Delta n=0.15$, after which the absorption saturates to its OFF-state value.

Cavity-induced enhancement and deep modulation of SERS. In a different application example, the enhanced field strengths associated with cavity-coupled plasmonics (Fig. 2b) can be exploited to improve performance in SERS-based sensors. To demonstrate this possibility, we examined scattering from a monolayer of benzenethiol formed on samples like the one of Figure 1c, using linearly polarized excitation light at $\lambda_{\mathrm{Ex}}=785 \mathrm{~nm}$. Figure $5 \mathrm{a}$ shows measured 'Raman counts' (Rc) for 3 cases, all with $L_{1}=850 \mathrm{~nm}$, but with 3 different values for $H_{\mathrm{BR}}: 0,15$ and $200 \mathrm{~nm}$. The spectra show expected signatures for benzenethiol, but with signal enhancements, defined as $\operatorname{Enh}\left(L_{1}, H_{\mathrm{BR}}\right)=\operatorname{Rc}\left(L_{1}, H_{\mathrm{BR}}\right) / \operatorname{Rc}\left(L_{1}, H_{\mathrm{BR}}=0\right)$, of $\sim 350 \%$ and $\sim 200 \%$ for the bands at 1,574 and $1,073 \mathrm{~cm}^{-1}$, respectively. This outcome is consistent with the physics summarized in Figure 2. Figure 5b shows the average intensity computed by FDTD on a conformal surface $10 \mathrm{~nm}$ above top and bottom Au layer for $H_{\mathrm{BR}}=200 \mathrm{~nm}$ and $L_{1}=850 \mathrm{~nm}$, where benzenethiol is considered as the surrounding medium. The dominant local maximum observed in this plot is identified as an LSPR. The wavelengths of the Raman peaks are $\lambda_{\text {Raman } 1}=857 \mathrm{~nm}$ and $\lambda_{\text {Raman2 }}=896 \mathrm{~nm}$. The LSPR resonance $\left(\lambda_{\mathrm{LSPR}}\right)$ spans across wavelengths that lie halfway between $\lambda_{\mathrm{Ex}}$ and $\lambda_{\text {Raman }}$, for both peaks, thereby simultaneously satisfying the approximate requirement $\left(\lambda_{\mathrm{LSPR}} \approx\left(\lambda_{\mathrm{Ex}}+\lambda_{\text {Raman }}\right) / 2\right)$ for maximum SERS enhancement ${ }^{1,28,29}$. Figure $5 \mathrm{c}$ shows the measured variation of enhanced Raman counts at $\lambda_{\text {Raman2 }}$, as a function of $H_{\mathrm{BR}}$, together with FDTD-computed values of normalized field intensity squared $\left(E_{\mathrm{Nor}}^{4}\right)$ where $E_{\mathrm{Nor}}=\left|E_{\mathrm{BR}}^{\text {ave }}\right| /\left|E_{\mathrm{BR}=0}^{\text {ave }}\right|$, spatially averaged at $10 \mathrm{~nm}$ away from the surfaces, as in the other cases, at a wavelength of $850 \mathrm{~nm}$, normalized to a structure with $H_{\mathrm{BR}}=0$, all with $L_{1}=850 \mathrm{~nm}$. The experimental and FDTD results show remarkably good agreement, thereby providing confirmation of the cavity-related physics and origins of SERS signals.

The plasmon resonances can be constructively or destructively influenced by cavity modes. Figure $5 \mathrm{~d}$ shows the result of this effect 

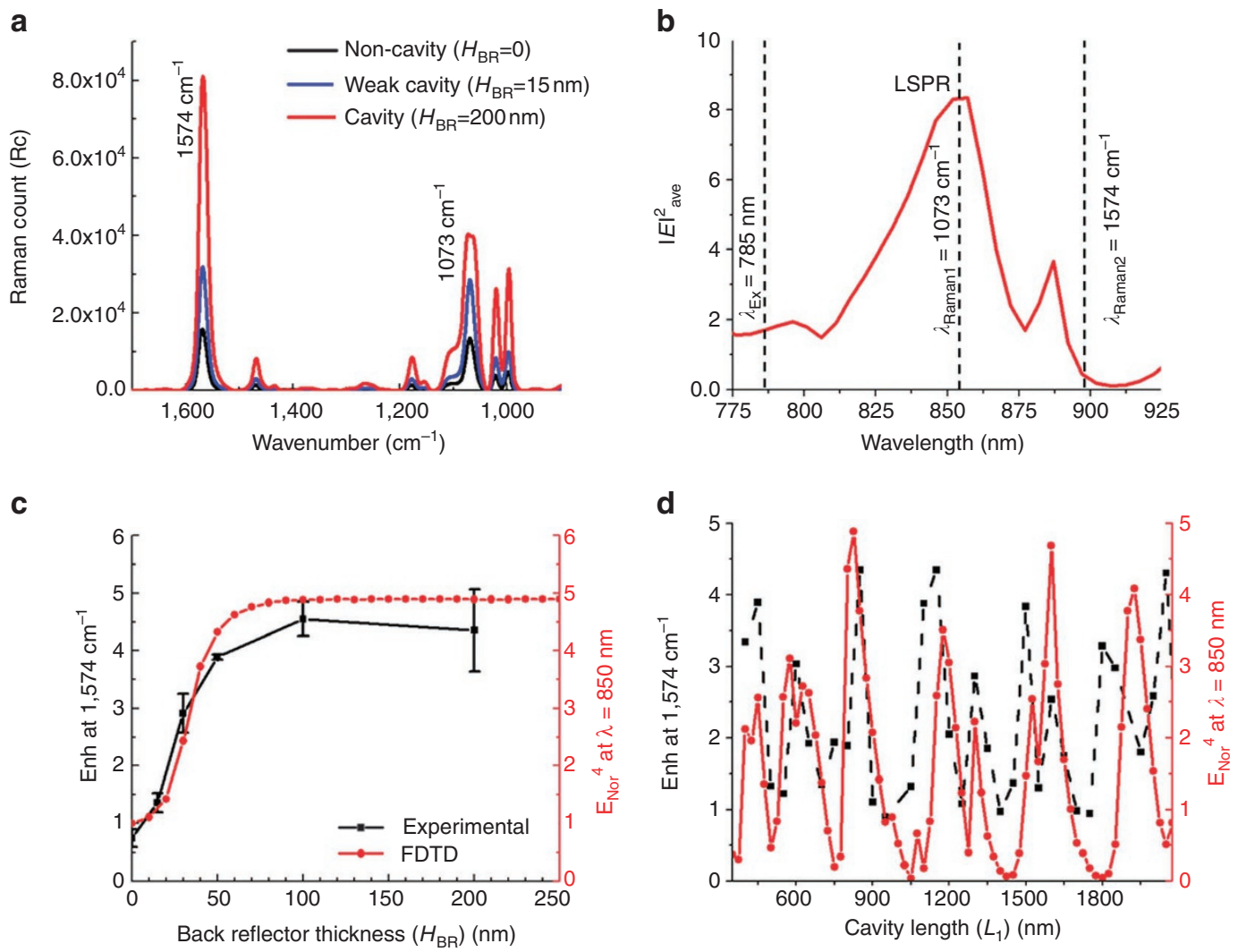

Figure 5 | Enhancement and modulation of Raman signal. (a) Measured Raman spectra for non-cavity $\left(H_{B R}=0\right)$ and cavity $\left(H_{B R}=15\right.$ and $\left.200 \mathrm{~nm}\right)$ configurations of quasi-3D plasmonic crystals. The two main peaks observed here, $\lambda_{\text {Raman1 }}$ and $\lambda_{\text {Raman2, }}$ are characteristic of benzenethiol. (b) Corresponding FDTD-computed intensity, $|E|_{\text {ave }}^{2}$ averaged across a conformal surface located $10 \mathrm{~nm}$ above the top and bottom Au layers, for a back reflector thickness of $H_{B R}=200 \mathrm{~nm}$ and cavity length $L_{1}=850 \mathrm{~nm}$ (all other parameters remained same). The dominant peak is due to an LSPR. The locations of the laser excitation and Raman peaks are marked as $\lambda_{\mathrm{Ex},} \lambda_{\text {Raman } 1}$ and $\lambda_{\text {Raman2 }}$ respectively. (c) Measured variation of the Raman enhancement at $\lambda_{\text {Raman2 }}=1,574 \mathrm{~cm}^{-1}$ and corresponding FDTD-computed normalized, and spatially averaged, squared intensity $\left(E_{\mathrm{Nor}}{ }^{4}\right)$, both as a function of back reflector thickness. (d) Plot similar to the one in (c), but plotted as a function of cavity length.

on the SERS measurement, in which the enhancement in Raman counts for $\lambda_{\text {Raman2 }}$ appears as a function of cavity length for constant $H_{\mathrm{BR}}=200 \mathrm{~nm}$. As with Figure $5 \mathrm{c}$, the Raman counts and FDTDcomputed average values of $|E|^{4}$, again normalized to the case where $H_{\mathrm{BR}}=0$, exhibit good agreement. The results show SERS signals that follow cavity-coupling effects discussed previously in the context of Figure $2 \mathrm{~b}$. The ability to control the response over a range of $>4 \times$, including strong amplification (that is, $350 \%$ increase) by adjusting only the cavity length is a notable outcome of the physics. Further optimized structures and materials offer promise for achieving even larger effects.

\section{Discussion}

This collective set of results indicates that simple strategies for coupling optical cavities to plasmonic modes can induce responses with practical interest in photonics and sensing. The out-of-plane cavities and the quasi-3D plasmonic crystals presented here are not the only types of structures that can be configured in this way. Arrays of metallic particles, waveguide resonators, in-plane cavities and many other possibilities also appear interesting to consider. A broad consideration of these layouts, together with other materials, in homogeneous or heterogeneous configurations, represent directions for future work.

\section{Methods}

Plasmonic cavity fabrication. Large area $(5 \mathrm{~mm} \times 5 \mathrm{~mm})$ square arrays of subwavelength cylindrical depressions were moulded onto the surface of a thin layer of a photo curable epoxy (SU8, MicroChem), using the techniques of solvent-assisted soft imprint lithography, wherein a small amount of ethanol was used to wet the epoxy surface before pressing the substrate against a poly(dimethylsiloxane) (PDMS, 10:1 Sylgard 184) mould containing the inverse pattern. The thickness of the epoxy was controlled through dilution of SU8 photoresist in cyclopentanol and conditions for the spin-coating process. A gold-coated glass substrate served as the substrate. Blanket deposition of a thin layer of gold $(\sim 50 \mathrm{~nm})$ by electron beam evaporation completed the fabrication process.

Opto-fluidic system. The opto-fluidic system was created with a piece of a PDMS with suitable structures of relief on its surface, formed by casting and curing against a pattern of photoresist on a silicon wafer. Contacting the PDMS against a substrate that supports a cavity-coupled plasmonic crystal led to the formation of a liquid-tight seal, thereby establishing a microfluidic channel. Liquid was injected into the channel, through tubes inserted into inlets machined through the thickness of the PDMS, using a syringe pump. For refractive index sensing, we used ethanol $(n=1.3614)$, tetrahydrofuran $(n=1.4072)$, chloroform $(n=1.4458)$ and toluene $(n=1.4969)$.

FDTD model. The detailed FDTD model of the actual structure of Figure 1e appears in Supplementary Figure S5 where Cavity (I) and Cavity (II) lengths are $L_{1}, L_{2}$ and the back reflector, top and bottom gold layer, relief depth, side wall height and thicknesses are $H_{\mathrm{BR}}, H_{\mathrm{top}}, H_{\text {bottom }}, H_{\mathrm{RD}}, H_{\text {sidewall }}$ and $t_{\text {sidewall }}$ respectively. A Drude plus two-pole Lorentzian model for the complex frequency-dependent gold permittivity was employed, with parameters fit to empirical dielectric constant data $^{30}$. The structure was excited with linearly polarized, plane-wave light. Here we used FDTD to 'fit' our transmission data as shown in Supplementary Figure S6, and then used that model, with those fitted parameters, to explore various trends and effects with cavity length, index and back reflector thicknesses. To establish correspondence between experiment and modelling (Supplementary

Fig. S5), transmission through two different geometries, one with no back reflector $\left(H_{\mathrm{BR}}=0\right)$ and one with a thin back reflector $\left(H_{\mathrm{BR}}=15 \mathrm{~nm}\right)$, were compared, both 
with $L_{1}=710 \mathrm{~nm}$ and $H_{\mathrm{RD}}=360 \mathrm{~nm}$. We then varied $H_{\text {top }}$ between $45-55 \mathrm{~nm}, H_{\text {bottom }}$ between $45-55 \mathrm{~nm}, H_{\text {sidewall }}$ between $90-120 \mathrm{~nm}$, and $t_{\text {sidewall }}$ between $10-25 \mathrm{~nm}$, in an iterative fashion, to yield a good match between experiment and modelling. The best fit was found for constant $L_{1}=710 \mathrm{~nm}, H_{\mathrm{RD}}=360 \mathrm{~nm}$ and $H_{\text {top }}=50 \mathrm{~nm}$, $H_{\text {bottom }}=40 \mathrm{~nm}, H_{\text {sidewall }}=100 \mathrm{~nm}$, and $t_{\text {sidewall }}=15 \mathrm{~nm}$ thicknesses. A final optimization was then performed by varying $L_{1}$ by $\pm 20 \mathrm{~nm}$ to yield a 'best' fit to study the effects of cavity length on LSPR keeping all other parameters constant. In all cases, the magnitudes of variations explored for each parameter were within uncertainties in physical measurements (ellipsometer, SEM). The transmission through the fabricated structure and FDTD model (Supplementary Fig. S5), for two different geometries, one non-cavity geometry having no back reflector $\left(H_{\mathrm{BR}}=0\right)$ and the other one with cavity geometry having a thin back reflector $\left(H_{\mathrm{BR}}=15 \mathrm{~nm}\right)$, were compared for constant $L_{1}=710 \mathrm{~nm}, H_{\mathrm{RD}}=360 \mathrm{~nm}, H_{\text {top }}=50 \mathrm{~nm}, H_{\text {bottom }}=40 \mathrm{~nm}$, $H_{\text {sidewall }}=100 \mathrm{~nm}$ and $t_{\text {sidewall }}=15 \mathrm{~nm}$. Supplementary Figure S6a,b shows experimental as well as FDTD-predicted 0-th order transmissions of these two structures, respectively. It can be observed that for the given set of experimentally measured thicknesses, FDTD predictions of transmissions through these two structures matches well with experimental observations. To emulate the actual SERS experimental procedures in FDTD simulation, a 5-nm thick Benzenethiol layer was created on nanohole surface and similar mapping of cavity length versus plasmon resonance was computed. As expected, we observed red-shift of plasmon resonances when the sample is coated with Benzenethiol having higher refractive index $(n=1.588)$ than air.

Far-field reflection measurements. Reflection spectra were collected using a $\times 2.5,0.07$ numerical objective on an optical microscope (Hyperion 2000) coupled to a Fourier transform infrared spectrometer (Vertex 70) and outfitted with a spatial aperture having a diameter of $3.75 \mathrm{~mm}$. Reflection spectra were normalized to a silver mirror with $96 \%$ reflectivity over the wavelengths examined.

Raman measurements. Raman spectra were acquired using a confocal Raman microscope (Senterra, Bruker Optics) with a $75-\mathrm{mm}$ focal-length achromatic lens for both illumination and collection, in place of a microscope objective. The diameter of the pinhole aperture was set to $50 \mu \mathrm{m}$. For all measurements, the spectral region from $400-1,800 \mathrm{~cm}^{-1}$ was collected with $3-5 \mathrm{~cm}^{-1}$ spectral resolution. The laser power at the sample was $33 \mathrm{~mW}$ with a spot size of $95 \mu \mathrm{m}$. The acquisition times were $30 \mathrm{~s}$ for all measurements. The sample was illuminated with linearly polarized laser light with a wavelength of $785 \mathrm{~nm}$. The laser beam filled an $2 \mathrm{~mm}$ region on the back aperture of the achromatic lens, corresponding to an angular incidence of illumination with a numerical aperture of $\sim 0.02$. Data processing of Raman spectra was achieved using OPUS 6.5 (Bruker), including background corrections to account for the dark current response and quantum efficiency of the chargecoupled detector device. Wavelength calibration was also accomplished using OPUS 6.5 to adjust the Raman shift using the atomic emission spectrum of mixed neon and argon gases.

\section{References}

1. Maier, S. A. Plasmonics: Fundamentals and Applications (Springer, 2007).

2. Homola, J. Surface plasmon resonance sensors for detection of chemical and biological species. Chem. Rev. 108, 462-493 (2008).

3. Bozhevolnyi, S. I., Volkov, V. S., Devaux, E., Laluet, J. Y. \& Ebbesen, T. W. Channel plasmon subwavelength waveguide components including interferometers and ring resonators. Nature 440, 508-511 (2006).

4. Chen, L., Shakya, J. \& Lipson, M. Subwavelength confinement in an integrated metal slot waveguide on silicon. Opt. Lett. 31, 2133-2135 (2006).

5. Pacifici, D., Lezec, H. J. \& Atwater, H. A. All-optical modulation by plasmonic excitation of CdSe quantum dots. Nat. Photonics 1, 402-406 (2007).

6. Ditlbacher, H., Krenn, J. R., Schider, G., Leitner, A. \& Aussenegg, F. R. Twodimensional optics with surface plasmon polaritons. Appl. Phys. Lett. 81, 1762-1764 (2002)

7. Tang, L., Latif, S. \& Miller, D. A. B. Plasmonic device in silicon CMOS. Electron. Lett. 45, 706-708 (2009).

8. Rosenberg, S., Shenoi, R. V., Vandervelde, T. E., Krishna, S. \& Painter, O. A multispectral and polarization-selective surface-plasmon resonant midinfrared detector. Appl. Phys. Lett. 95, 161101 (2009).

9. Perahia, R., Alegre, T. P. M., Safavi-Naeini, A. H. \& Painter, O. Surface-plasmon mode hybridization in subwavelength microdisk lasers. Appl. Phys. Lett. 95, 201114 (2009)

10. Atwater, H. A. \& Polman, A. B. Plasmonics for improved photovoltaic devices. Nat. Mat. 9, 205-213 (2006).
11. Lassiter, J. B. et al. Fano resonances in plasmonic nanoclusters: geometrical and chemical tunability. Nano Lett. 10, 3184-3189 (2010).

12. Fan, J. A. et al. Self-assembled plasmonic nanoparticle clusters. Science 328, 1135-1138 (2010).

13. Langhammer, C., Schwind, M., Kasemo, B. \& Zorić, I. Localized surface plasmon resonances in aluminum nanodisks. Nano Lett. 8, 1461-1471 (2008).

14. Sherry, L. J., Chang, S., Schatz, G. C. \& Duyne, R. P. V. Localized surface plasmon resonance spectroscopy of single silver nanocubes. Nano Lett. 5, 2034-2038 (2005).

15. Stewart, M. E. et al. Nanostructured plasmonic sensors. Chem. Rev. 108, 494-521 (2008).

16. Choi, Y., Choi, D. \& Lee, L. P. Metal-insulator-metal optical nanoantenna with equivalent-circuit analysis. Adv. Mater. 22, 1754-1758 (2010).

17. Xu, T., Wu, Y., Luo, X. \& Guo, L. J. Plasmonic nanoresonators for highresolution colour filtering and spectral imaging. Nat. Commun. 1, 59 (2010).

18. Diest, K., Dionne, J. A., Spain, M. \& Atwater, H. A. Tunable color filters based on metal-insulator-metal resonators. Nano Lett. 9, 2579-2583 (2009).

19. Malyarchuk, V. et al. High performance plasmonic crystal sensor formed by soft nanoimprint lithography. Opt. Express 13, 5669-5675 (2005).

20. Baca, A. J. et al. Molded plasmonic crystals for detecting and spatially imaging surface bound species by surface-enhanced Raman scattering. Appl. Phys. Lett. 94, 243109 (2009).

21. Lee, M. H., Huntington, M. D., Zhou, W., Yang, J. \& Odom, T. W. Programmable soft lithography: solvent-assisted nanoscale embossing. Nano Lett. 11, 311-315 (2010).

22. Stewart, M. E. et al. Quantitative multispectral biosensing and $1 \mathrm{D}$ imaging using quasi-3D plasmonic crystals. Proc. Natl Acad. Sci. USA 103, 17143-17148 (2006)

23. Landy, N. I., Sajuyigbe, S., Mock, J. J., Smith, D. R. \& Padilla, W. J. Perfect metamaterial absorber. Phys. Rev. Lett. 100, 207402 (2008)

24. Kravets, V. G., Schedin, F. \& Grigorenko, A. N. Plasmonic blackbody: almost complete absorption of light in nanostructured metallic coatings. Phys. Rev. B 78, 205405 (2008).

25. Hu, C., Zhao, Z., Chen, X. \& Luo, X. Realizing near-perfect absorption at visible frequencies. Opt. Express 17, 11039-11044 (2009).

26. Yariv, A. \& Pochi, Y. Photonics: Optical Electronics in Modern Communications, 6th edn (Oxford University Press, 2007).

27. Geis, W. et al. Fabrication of crystalline organic waveguides with an exceptionally large electro-optic coefficient. Appl. Phys. Lett. 84, 3729-3731 (2004).

28. Reilly, T. H., Chang, S., Corbman, J. D., Schatz, G. C. \& Rowlen, K. L. Quantitative evaluation of plasmon enhanced Raman scattering from nanoaperture arrays. J. Phys. Chem. C 111, 1689-1694 (2007).

29. Chu, Y., Banaee, M. G. \& Crozier, K. B. Double-resonance plasmon substrates for surface-enhanced Raman scattering with enhancement at excitation and stokes frequencies. ACS Nano 4, 2804-2810 (2010).

30. Gray, S. K. \& Kupka, T. Propagation of light in metallic nanowire arrays: finite-difference time-domain studies of silver cylinders. Phys. Rev. B 68, 045415 (2003).

\section{Acknowledgements}

The work at University of Illinois was supported by a grant from the Office of Naval Research. We thank Audrey M. Bowen for providing photoresist masters for the opto-fluidic chips.

\section{Author contributions}

D.C. conceived and designed the experiments. D.C., K.S., T. T and E.L. performed the experiments. D.C. analysed and simulated the data. A.M., M.S., P.B. and R.B. contributed materials/analysis tools. J.A.R. provided guidance. D.C. and J.A.R. co-wrote the paper.

\section{Additional information}

Supplementary Information accompanies this paper at http://www.nature.com/ naturecommunications

Competing financial interests: The authors declare no competing financial interests.

Reprints and permission information is available online at http://npg.nature.com/ reprintsandpermissions/

How to cite this article: Chanda, D. et al. Coupling of plasmonic and optical cavity modes in quasi-three-dimensional plasmonic crystals. Nat. Commun. 2:479 doi: 10.1038/ncomms1487 (2011) 